\title{
Process Design of Laser Powder Bed Fusion of Stainless Steel Using a Gaussian Process Based Machine Learning Model
}

\author{
Lingbin Meng, Jing Zhang* \\ Department of Mechanical and Energy Engineering \\ Indiana University-Purdue University Indianapolis, Indianapolis, IN 46202, USA \\ *Corresponding author: jz29@iupui.edu
}

\begin{abstract}
In this work, a Gaussian process (GP) based machine learning model is developed to predict the remelted depth of single tracks, as a function of combined laser power and laser scan speed in laser powder bed fusion (L-PBF) process. The GP model is trained by both simulation and experimental data from the literature. The mean absolute prediction error magnified by the GP model is only $0.6 \mu \mathrm{m}$ for a powder bed with layer thickness of $30 \mu \mathrm{m}$, suggesting the adequacy of the GP model. Then the process design maps of two metals, 316L and 17-4 PH stainless steel, are developed using the trained model. The normalized enthalpy criterion of identifying keyhole mode is evaluated for both stainless steel. For $316 \mathrm{~L}$, the result suggests that the $\frac{\Delta H}{h_{s}} \geq 30$ criterion should be related to the powder layer thickness. For 17-4 PH, the criterion should be revised to $\frac{\Delta H}{h_{s}} \geq 25$.
\end{abstract}

Keywords: additive manufacturing; Gaussian process; machine learning; powder bed fusion

This is the author's manuscript of the article published in final edited form as: 


\section{Introduction}

Additive manufacturing (AM) is defined as a group of layer-upon-layer fabrication processes and previously applied for rapid prototyping. Laser powder bed fusion (L-PBF) is an AM technique that uses a laser as the power source to selectively melt or sinter a metallic powder bed. Among AM techniques, L-PBF process tends to be the most common for metallic materials, due to their capability of producing parts with improved density, resolution, and surface finish that require less post-processing compared to other processes such as binder jetting [1]. One of the major challenges in L-PBF technique is defect formation in printed products. The quality of the fused powder is affected by numerous processing parameters, including laser power, laser scan speed, laser beam size, powder size, layer thickness, and material absorptivity, especially those parameters related to energy input.

In laser processing field, there are two modes of heat transfer mechanisms related to defect formation: (1) conduction mode, in which the melting is controlled by heat conduction, and (2) keyhole mode, in which the input power density of the laser beam is sufficient to vaporize the metal and drill a much deeper cavity than in conduction mode [2]. Since the collapse of the cavities in keyhole mode may leave voids in the printed parts [3], the conduction mode is preferred in laser additive manufacturing. Therefore, for L-PBF process, if the processing parameters are not well tuned, the energy input can be either insufficient to cause lack of fusion issue, or overlarge to cause pores formation in keyhole mode. Consequently, the understanding of processing parameters in L-PBF is critical to for a robust design.

Forerunners have made lots of efforts in process optimization by experimental [4-8] and computational study [9-16]. For designers, on one hand, experiments and testing are helpful to determine the preferred conduction-controlled region of processing parameters, but they are also time-consuming and expensive. On the other hand, the modeling and simulation tools enable designers to make predictions of the printed products of L-PBF so as to optimize the processing parameters and decrease the need for conducting experiments. In addition, those mesoscopic simulation models are extremely helpful in understanding the physical meaning of the process. In spite of these advantages, models with high accuracy are usually computationally intensive. It is sometimes impractical to optimize the processing parameters using either experimental or 
computational study, especially when the number of involved processing parameters is large. Consequently, there is a need of a more efficient way for process design.

An attractive alternative to this dilemma is the application of the data-driven tool, e.g., machine learning (ML) models. Machine learning can be defined as computer programming to optimize a performance criterion using example data or past experience [17]. In general, ML tasks can be divided into three groups, supervised learning, unsupervised learning, and reinforcement learning [17]. In AM field, the type of applied ML technique is usually the supervised learning, in which each input datum is labeled with an output, and predictions are made from an unobserved input to an output based on example input-output pairs. The input is a vector contains all involved processing parameters. The output can be either classes and categories such as defect types and the corresponding ML task is classification, or parameters such as porosity and the corresponding ML task is regression. With a reliable training dataset, the ML models learn knowledge from the training set and make inference based on the knowledge. After training, ML models can make prediction at any new input points without making more observations, and thus reduce the need of experimental and computational study. In this work, the remelted depth will be used as a pointer of the quality of the printed parts, as it indicates how well the successive layers bond, whether the energy from the laser is sufficient, and whether the keyhole mode occurs. As the remelted depth, the output in this work, is a parameter, this work focuses on regression ML models. In a word, the objective of this work is to develop an efficient ML based method to assist process design and optimization.

Pioneers have applied supervised machine learning models to various AM regression tasks. Z. Zhu et al. [18] developed a Gaussian process (GP) regression model to make predictions on shape deviation based on the input geometry with an average R-square of around 0.97. G. Tapia et al. [19] developed a GP regression model to makes predictions using the experimental data of 17-4 PH stainless steel, and demonstrated how to determine the optimal processing parameters at desired porosity using GP model trained by an experimental dataset with high noise level. G. Tapia et al. [20] also applied the GP model to make predictions of remelted depth based on laser power and laser scan speed using experimental data of 316L stainless steel. Their model predicts consistent processing windows with the normalized enthalpy $>30$ criterion to identify keyhole 
mode in L-PBF [6], which confirms the validation of their model. In these pioneers' efforts, GP shows its excellent regression performance in AM field. Neural network (NN), on the other hand, also plays a role in regression tasks in AM field. Caiazzo et al. [21] developed a NN regression model for trace geometry prediction and the root mean square errors (RMSE) is around 5\%. Zhang et al. [22] developed a recurrent NN model in material extrusion process to predict the tensile strength of the printed products and the RMSE was around 2\%. Mozaffar et al. [23] developed a recurrent NN model to predict the thermal history in directed energy deposition processes and achieved a mean square error of $2.97 \times 10^{-5}$. Overall, $\mathrm{NN}$ is a powerful model in regression tasks in AM field, but it also requires tuning a series of hyperparameters such as number of hidden neurons and layers [24], which makes NN complicated to apply. Therefore, GP will be applied as the regression model in this work.

In this work, a Gaussian process regression model is developed to assist the process design and optimization of L-PBF process. The whole paper is divided into two parts. The first part, Section 2 and Section 3, focuses on developing and validating an efficient GP regression model which can generate a regression surface based on a training dataset and make predictions at any input settings. To obtain the training dataset, a computational fluid dynamics (CFD) model is applied to perform a set of single track simulations of the melting process of laser powder bed fused $316 \mathrm{~L}$ stainless steel. The remelted depth is then measured from the simulations at various combinations of laser scan speed $(v)$ and laser power $(P)$, which forms the training dataset. Subsequently, a GP regression model is developed to generate a regression surface to make predictions at the unobserved settings based on observed data from CFD. The predictions are compared against experimental data. The second part, Section 4, demonstrates the applications of machine learning models in process design. Two datasets of experimental observations of $316 \mathrm{~L}$ and 17-4 PH stainless steel are used to train the GP model. Then, the conduction mode regions of both materials on the plane of laser scan speed and laser power are predicted and compared against the regions computed by normalized enthalpy. The criteria for identifying keyhole mode for different materials can be obtained by this comparison. Designers can make use of these predicted criteria to optimize the processing parameters, thus reducing the need for experimental and computational observations. 


\section{Methodology}

\subsection{Gaussian process regression model}

A Gaussian process is defined as a collection of random variables, such that any finite number of which has a joint Gaussian distribution [25]. In this work, the GP is applied as a regression method, which is different from an interpolation method. A regression task is aiming at finding a regularized way to fit a certain dataset so as to make better predictions for future data, whereas an interpolation task is aiming at perfectly fitting the certain dataset regardless of whether it predicts well or bad for future data [17].

Similar to the mean value and variance in Gaussian distribution, a GP is completely specified by a mean function $m\left(\boldsymbol{X}_{i}\right)$ and a covariance function $C\left(\boldsymbol{X}_{i}, \boldsymbol{X}_{j}\right)$, where $i$ and $j$ range from 1 to $n, n$ being the number of observation points. $\boldsymbol{X}$ is the input vector containing all processing parameters, and $\boldsymbol{X}=(P, v)$.

In this work, the GP model is expressed as:

$$
f(\boldsymbol{X})=G P\left(m\left(\boldsymbol{X}_{i}\right), C\left(\boldsymbol{X}_{i}, \boldsymbol{X}_{j}\right)\right)
$$

The statistical model can then be defined as:

$$
Y(\boldsymbol{X})=f(\boldsymbol{X})+e(\boldsymbol{X})
$$

where $Y$ is the output parameter of interest (i.e., the remelted depth $d$ in this work), and $e$ models the observation error which captures the inherent noise associated with the measurements. This observation error is usually treated as an independent term to the input vectors and represented by a Gaussian distribution with zero mean, i.e., $e \sim \mathcal{N}\left(0, \sigma^{2}\right)$. In a CFD model, no random seed is involved, which means that the measured remelted depth remains unchanged no matter how many simulations are conducted in the same setting. Thus, this error term can be disregarded. This omission will be verified by the fact that the noise level term approaches 0 after optimization in the result section.

In GP, the covariance function is defined by a single or a combination of kernel functions, which capture the spatial dependence between two different locations, $\boldsymbol{X}_{i}$ and $\boldsymbol{X}_{j}$. The selection of kernel functions should be based on practical applications, and has been discussed in Ref. [25]. In this work, the two input dimensions, $P$ and $v$, of the input vector $\boldsymbol{X}$ possess different units. 
Therefore, the Matern 5/2 kernel is employed [25], which allows different lengthscales for different input dimensions. The white-noise regularization is also added to the covariance function, in order to capture the noise level. As a result, 4 kernel hyperparameters are involved in this GP model, including the variances of the two kernel functions and the lengthscales of the two input dimensions. The initial values of both variances are set as 1 . The lengthscales of the laser scan speed and the laser power are reasonably set to be the mesh size, (i.e. 0.04, 10), respectively. These four hyperparameters are updated once a new observation is made and converge to the values that better predict future data. The GP regression model is performed using the GPy software package [26].

The performance of the GP model is quantitatively assessed by mean absolute prediction error (MAPE), which is defined as:

$$
\text { MAPE }=\frac{1}{n} \sum_{i=1}^{n}\left|Y_{o b s}\left(P_{i}, v_{i}\right)-Y_{\text {pre }}\left(P_{i}, v_{i}\right)\right|,
$$

where $n$ is the number of observation points from the CFD model.

\subsection{Simulation of melting process using computational fluid dynamics model}

A coupled solid-fluid-thermal model [16] is applied to simulate the melting process of laser powder bed fused 316L stainless steel. In this model, the initial configuration of the powder bed is generated using the discrete element method (DEM) [11]. A laser heat source is applied to the top surface of the powder bed, moving along $x$-direction in order to make a single track. The power of the laser heat source is represented by the Gaussian distribution [16]:

$$
S_{\text {laser }}=\frac{\alpha P}{\pi r_{0}{ }^{2}} \exp \left\{-\frac{2\left[(x-v t-i)^{2}+\left(y-y_{i}\right)^{2}\right]}{r_{0}{ }^{2}}\right\},
$$

where $P$ is the laser power, $\alpha$ is the absorption coefficient of the material, $r_{0}$ is the half of the laser beam diameter $\mathrm{D} 4 \sigma$ (D $4 \sigma$ is the beam diameter expressed by four times the standard deviation of the Gaussian distribution, and will be expressed as $\sigma$ thereafter), $v$ is the laser scan speed, and $x_{i}, y_{i}$ is the initial position of the laser focal center. The laser power is projected to the

top surface of the metal powders, such that all the heat source is absorbed by the top surface at the first interaction [27]. This laser model provides more physical phenomena, such as shadowing, than the volumetric heat source method. The reflection is ignored, a reasonable assumption as discussed in Ref. [16]. 
In this model, the conservation of mass, momentum, energy and volume is coupled with some complicated physical phenomena, including melting, buoyancy-driven flow, surface tension, Marangoni convection, and metal evaporation induced recoil pressure [16]. These conservation equations are modeled in User Defined Functions (UDF) in ANSYS Fluent. The laminar solver is applied with the assumption that the Reynolds number within the melt pool is small enough [28-30]. The mesh size is set as $3 \mu \mathrm{m}$ [16]. This CFD model is validated in Ref. [16] by comparing the simulation results with experimental results at the same settings.

In this work, a set of simulations using the CFD model is conducted to generate an observation dataset, $\boldsymbol{Y}_{o b s}(P, v)$, where $\boldsymbol{Y}_{o b s}$ is the predicted remelted depth as a function of laser power $P$ and laser scan speed $v$. The layer thickness and the laser beam diameter $\sigma$ keeps constant as 50 $\mu \mathrm{m}$ and $70 \mu \mathrm{m}$, respectively, so as to be consistent with the experimental settings [31]. The remelted depth for a single track is defined as $d$ in Fig.1. These observation points are then imported to the GP regression model as training data and validation data. Typically, the simulation time of a $1 \mathrm{~mm}$ single track simulation will be more than 20 hours in an Intel Core i7$8750 \mathrm{H}$ processor with 32 GB RAM [16]. The computational costs also reveal the necessity of the application of machine learning models to the process design and optimization. It is noted that the simulation model must been validated by either experiments or other models (which is in our case) before used for GP training later.

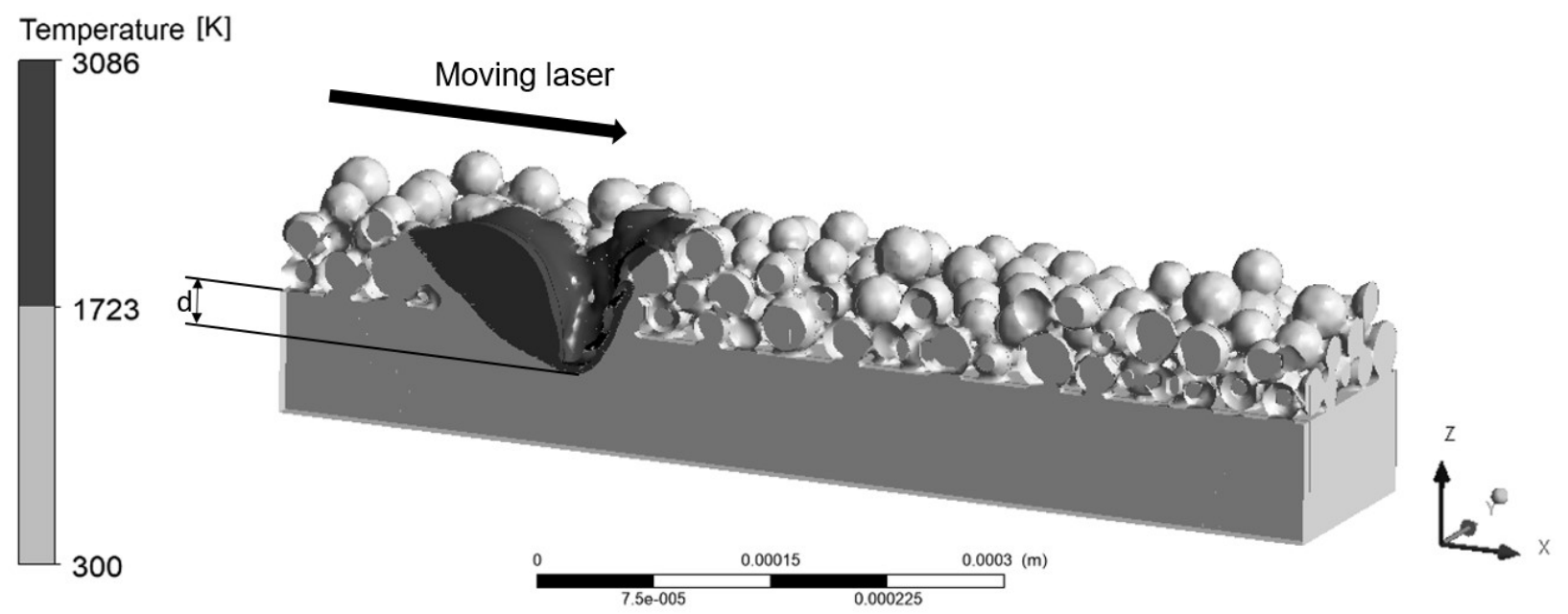


Fig. 1. Remelted region (black) is shown in the temperature contour of the CFD model cut through view at the plane $\mathrm{y}=0.1723 \mathrm{~K}$ is the liquidus of $316 \mathrm{~L}$ stainless steel. The remelted depth $d$ is illustrated as the average distance between the deepest melted region and the interface of the solid substrate and metal powders during the laser scanning.

\subsection{Validation using $\boldsymbol{n}$-fold cross-validation method}

Although the MAPE is able to quantitatively assess the performance of the machine learning model, a cross-validation method is still necessary because the MAPE will be always nearly 0 and make no sense if the GP model is trained by all data in a noiseless dataset. This is a characteristic of the GP models. In general, to assess the performance of a supervised learning model, the entire dataset is usually partitioned into at least two subsets, training set and testing set. The training set is used to optimize the hyperparameters of the model and make predictions at the same input settings of the testing data. The data in testing set will then be compared against the model predictions. If the model predictions are consistent with the testing set, then it confirms the validation of the machine learning model. In our case, the number of observation points is limited, as the CFD model is computationally expensive. Besides, a general trend of machine learning models is that the predictive performance has a positive correlation with the number of training data. Therefore, to make the utmost use of the limited data, the $n$-fold crossvalidation method is employed in this work.

The $n$-fold cross-validation, also called leave-one-out cross-validation (LOOCV), is an iterative procedure which can avoid overfitting issue and maximize the utilization of observation points. This is a special case of the widely known $k$-fold cross-validation method. In a $k$-fold crossvalidation process, the entire dataset is randomly separated into $k$ subsets of roughly same size. In each iteration, one subset is left out as the testing set and all other subsets form the training set. This iteration is repeated until all subsets have been used as the testing set once. The $n$-fold cross-validation is the case when $k$ is set as the number of observation points, or $k=n$. By this means, all data are fully utilized, and the intrinsic overfitting issue of GP is also resolved.

In this work, each GP prediction at a certain input setting, $\boldsymbol{X}_{i}=\left(P_{i}, v_{i}\right)$, is made by the machine trained by all data except $\boldsymbol{X}_{i}$. Fig. S1 in the Online Resource Electronic Supplementary Materials 
shows the flow chart of our GP model with the $n$-fold cross-validation method, where $n$ is the number of CFD observation points. These GP cross-predictions are compared against both the CFD results and experimental data. The difference between the MAPEs of these two comparisons will be the major performance indicator of our model, as it captures the error magnified by the Gaussian process model.

\section{Gaussian process model validation and results}

\subsection{Computational fluid dynamics observations}

In this work, 24 observation points in total are made using the CFD model, with laser power ranging from $30 \mathrm{~W}$ to $60 \mathrm{~W}$, and laser scan speed from $0.08 \mathrm{~m} / \mathrm{s}$ to $0.28 \mathrm{~m} / \mathrm{s}$. The resultant remelted depth in each case is measured, as summarized in Table S1 in the Online Resource Electronic Supplementary Materials. Those data points with 0 remelted depth indicate that the melted region at this input setting doesn't get in touch with the previous layer. In other words, the energy input in these settings is insufficient to sinter different powder layers. These observation points are then imported into the GP regression model to generate a regression surface.

\subsection{Gaussian process regression surface}

Using the GP regression model in Section 2.1 and the 24 observations points in Section 3.1, a regression surface is generated, as shown in Fig. 2. This plot follows the general trend that the remelted depth is increasing with increased energy input. The updated Matern kernel variance, white kernel variance, laser scan speed lengthscale and laser power lengthscale are 1267.9, $3.5456 \times 10^{-211}, 0.23962$ and 28.517 , respectively. It can be verified that the white kernel variance, which captures the noise level of the input dataset, is approaching 0 after optimization. This is the aforementioned expected result that the noise level of the CFD predictions is nearly 0 and can be reasonably disregarded. 


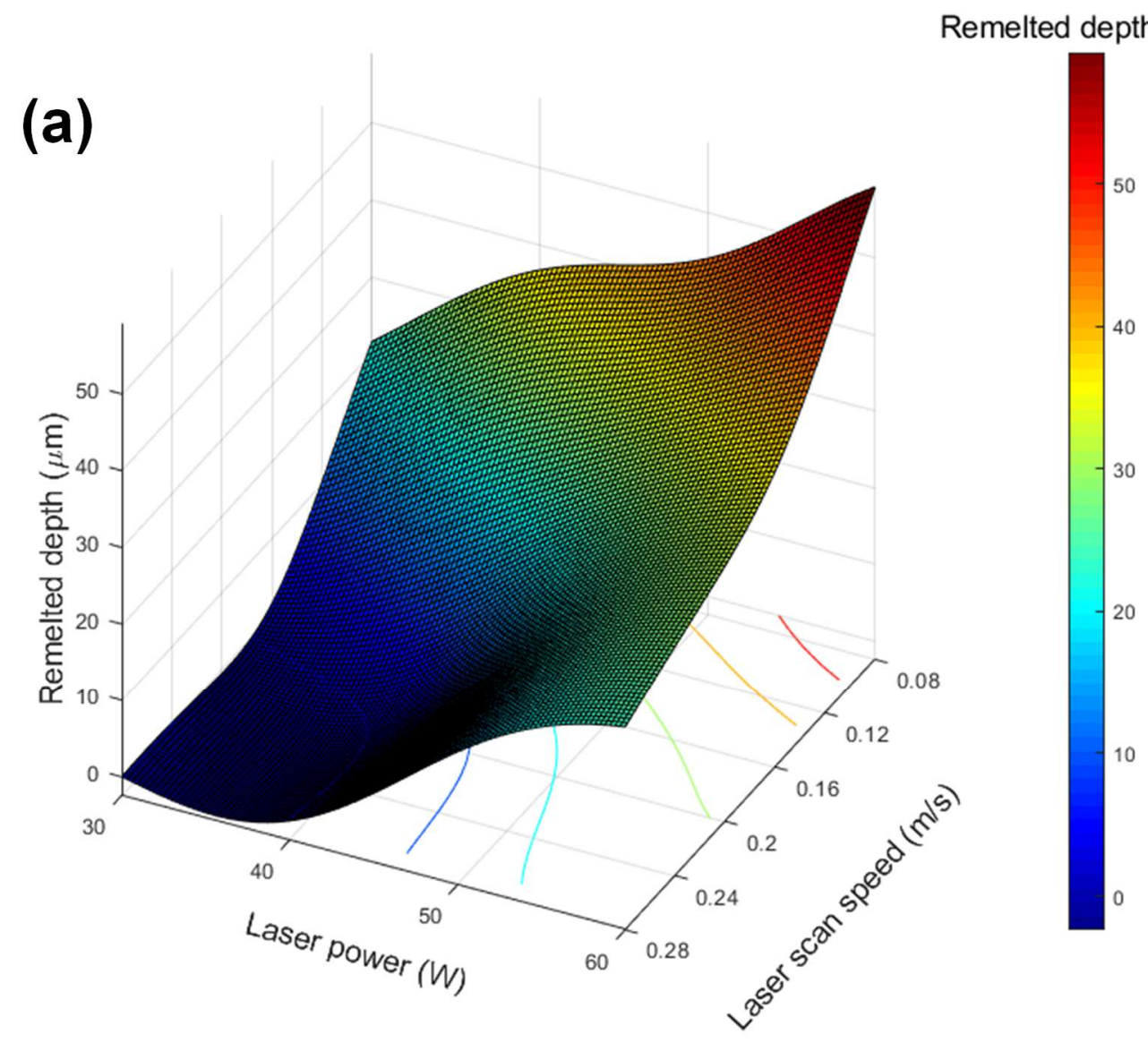




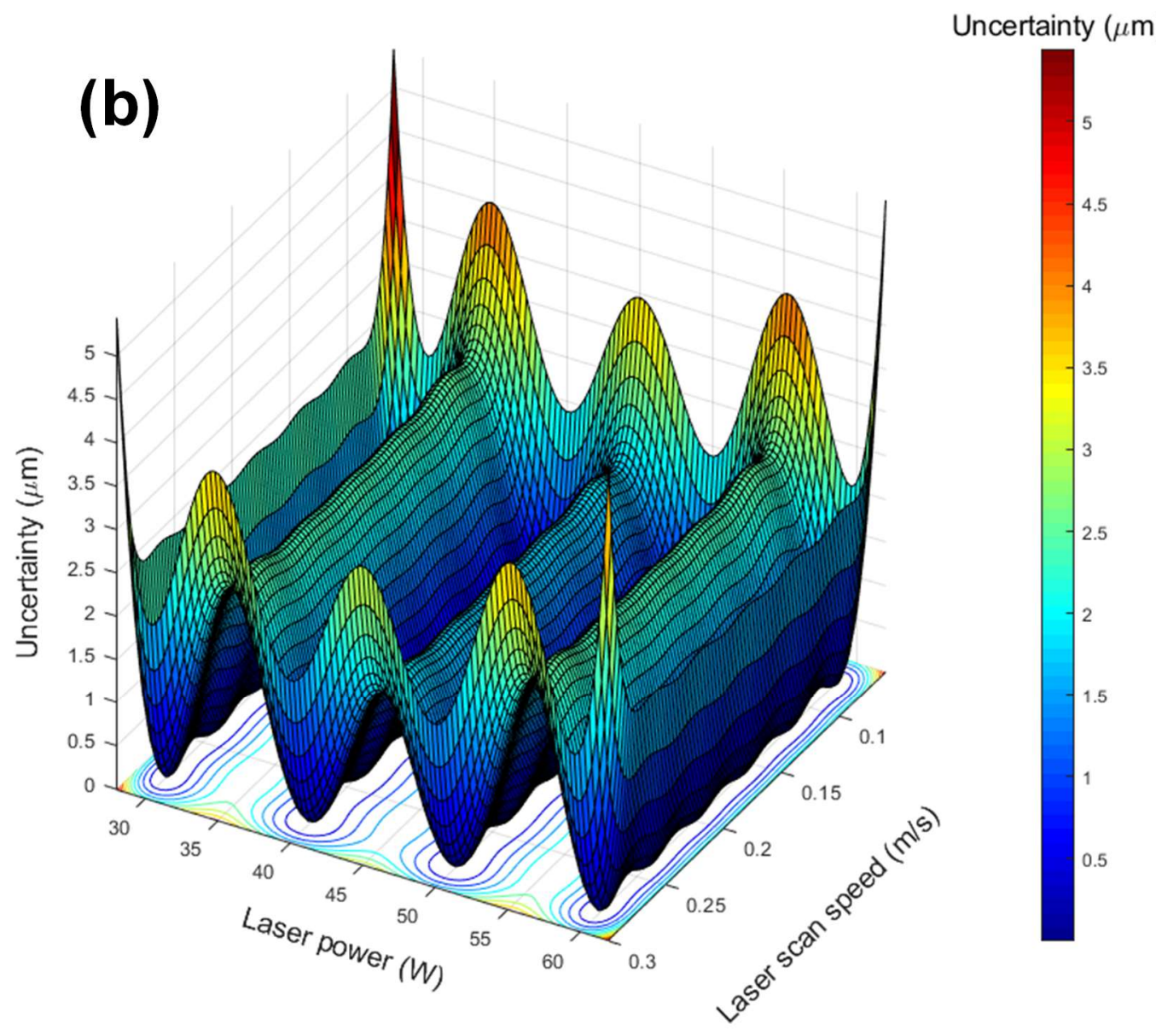

Fig. 2. (a) Regression surface of remelted depth as a function of laser power and scan speed made by the GP regression model, and (b) the corresponding uncertainty based on the $95 \%$ confidence interval. The grid in left figure represents the mesh of the data points from CFD.

The regression surface in Fig. 2 can then be used to predict the remelted depth with the corresponding uncertainty at any combinations of laser power and scan speed. Designers can make use of this process design map to predict the remelted depth at any settings without conducting more experimental or computational study. The uncertainty within the range of training dataset are relatively low, whereas the uncertainty outside the training range will be larger due to the extrapolation error. Since all 24 observation points are used to train the GP model and generate the regression surface, it can be observed that the uncertainty at the input settings of the 24 training points approach 0 . In other words, if the 24 training points are used again to test the performance of GP model, the model will make perfect predictions without any 
uncertainty. This is a characteristic of GP models in a noiseless environment. This exposes the significance of the cross-validation method.

\subsection{Cross-predictions and validations}

In this Section, the cross-validation method in Section 2.3 is employed. The $n$-fold crossvalidation plot is presented in Fig. 3. It represents a comparison between the cross-predictions made by the GP model and the CFD predictions. All data points are close to the ideal $\mathrm{y}=\mathrm{x}$ line, with MAPE $=1.4 \mu \mathrm{m}$. The low MAPE indicates the adequacy of the GP predictions. The total computational time for the whole iterative process is less than one minute, indicating the high efficiency of the GP model.

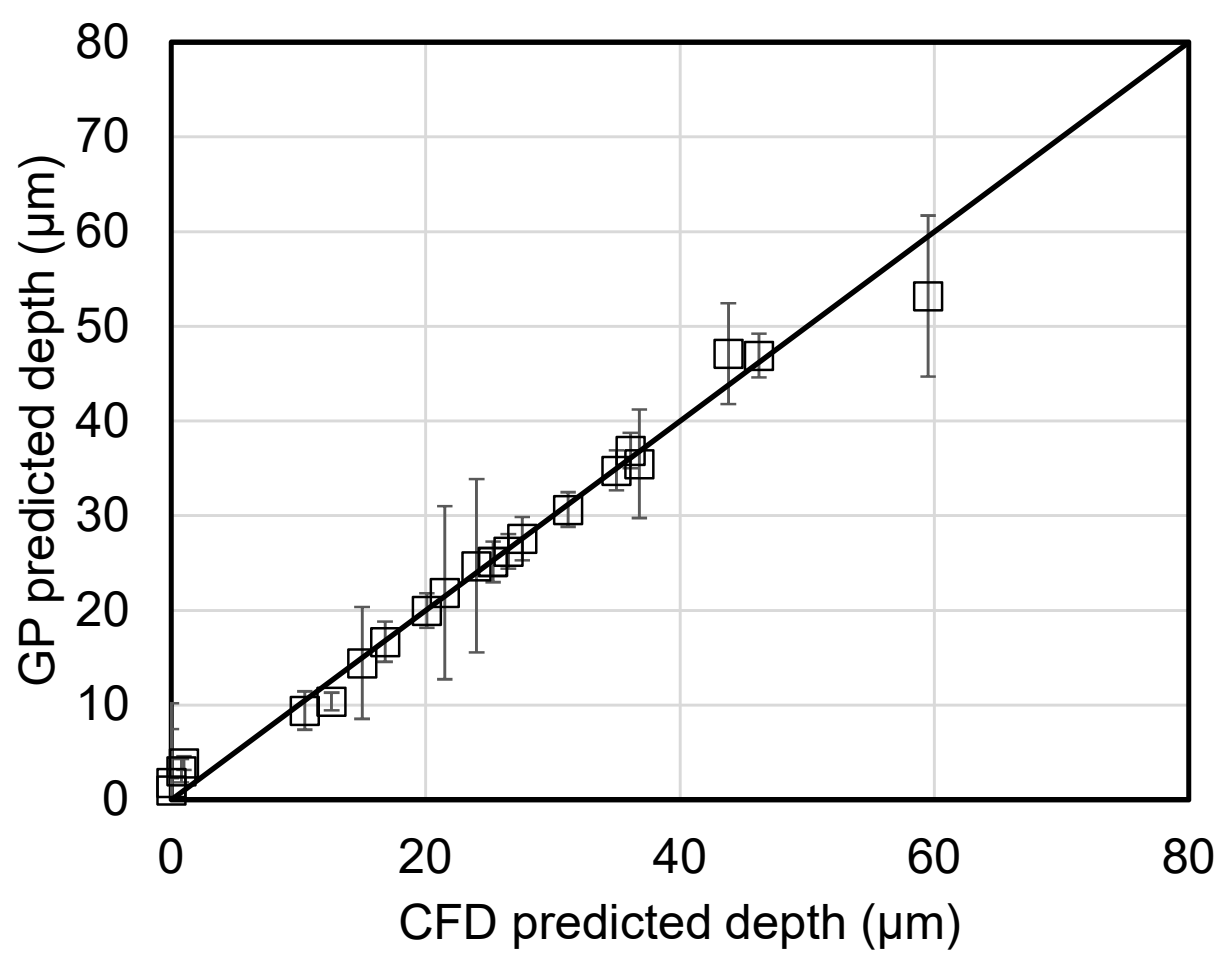

Fig. 3. Results of the $n$-fold cross-validation using the GP model and CFD model data

Up to now, the adequacy of the GP model in regression tasks in AM field are validated. To finally validate our model, the cross-predictions made by the GP model need to be compared against experimental results. This step is critical, because the GP regression model coupled with high-fidelity simulation models will become an effective tool for designers to reduce the need of 
expensive and time-consuming experiments. The validation plot is shown in Fig. 4, where the GP predicted depths are compared against experimentally observed depths. The quality of the predicted values is not as high as the CFD model as expected, but the GP model still provides a reasonable prediction.

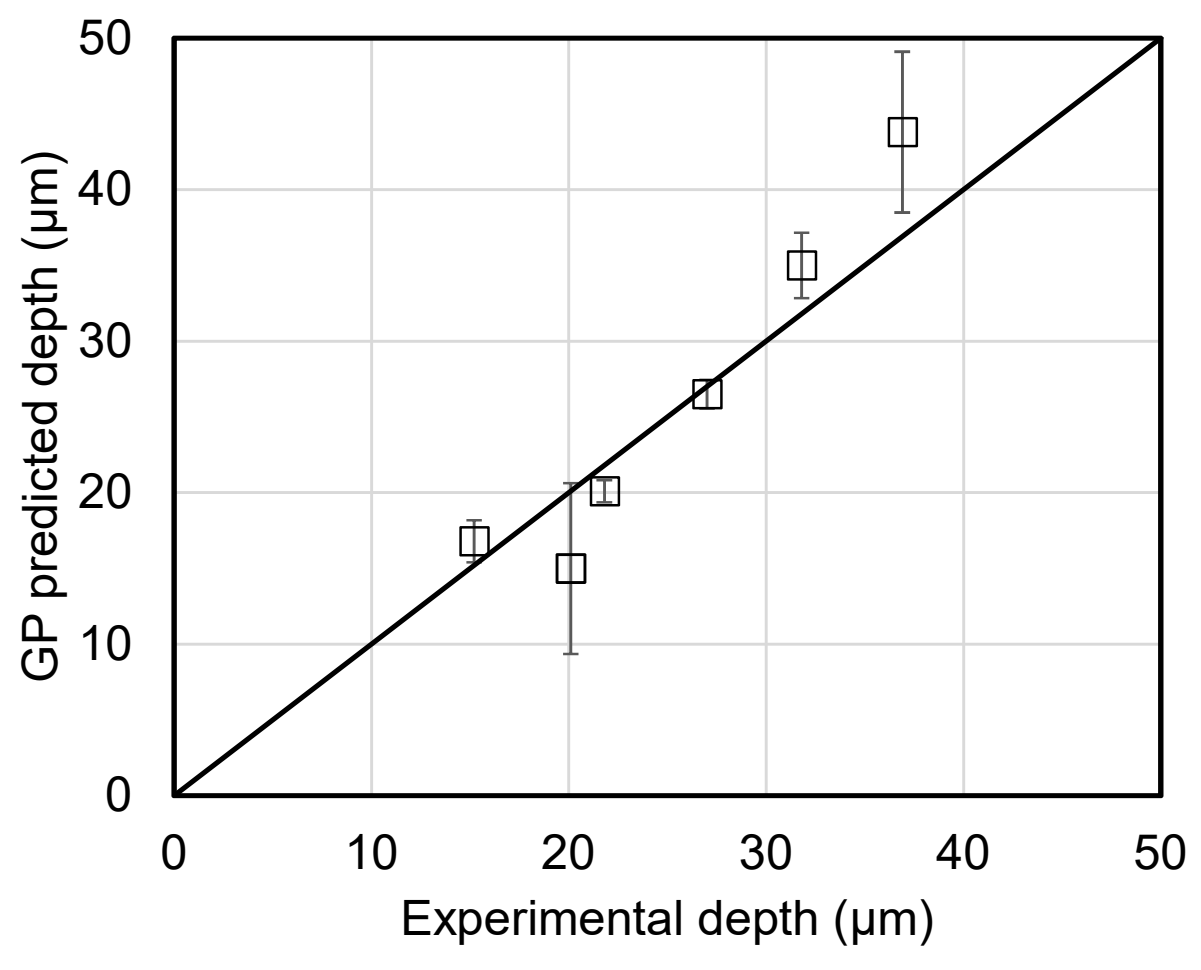

Fig. 4. Validation plot of GP predictions and experimental results

Fig. 5 shows the remelted depth comparison among the GP predictions, experimental results [31], and CFD predictions. For all the cases, with an increased speed and other parameters fixed, the energy input is decreased and should have led to a decreased remelted depth. In the experimental dataset, the laser scan speed is ranging from $0.08 \mathrm{~m} / \mathrm{s}$ to $0.28 \mathrm{~m} / \mathrm{s}$ with a fixed laser power of $50 \mathrm{~W}$. It should be noted that the noise level of the experimental dataset is not 0 , which can be observed between the data point of $0.24 \mathrm{~m} / \mathrm{s}$ and $0.28 \mathrm{~m} / \mathrm{s}$. 


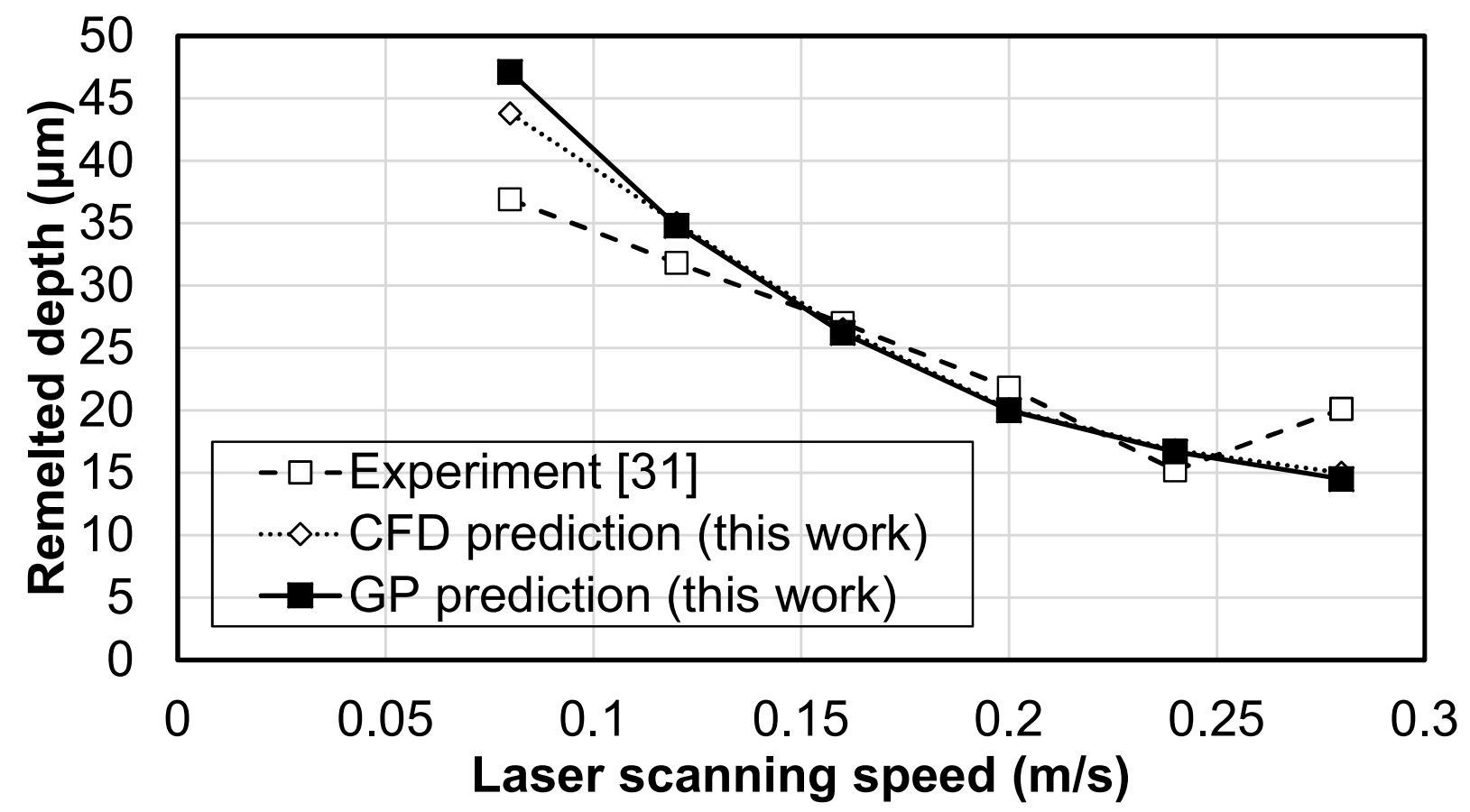

Fig. 5. Comparison of the remelted depth among experimental results [31], CFD prediction, and GP prediction.

In terms of the performance of the GP model, the MAPE between the GP predictions and experimental data is $3.8 \mu \mathrm{m}$, whereas the MAPE between the CFD predictions and experimental data is $3.2 \mu \mathrm{m}$. Since the GP model is trained by CFD predictions, the fact that the MAPE is only magnified by $0.6 \mu \mathrm{m}$, indicating the excellent performance of the GP model. It is worth mentioning that those data points with large error bars are all lying between the boundaries of the input range. The large standard variations are due to the extrapolation errors of the GP regression model. It can be avoided by conducting additional observations until these data points of interest are not lying at the boundary. Overall, the GP predictions are in good agreement with the experimental results. This confirms that the GP model developed in this work is capable of generalizing the predictions made by the CFD simulations to any other processing parameter settings within the predefined design space.

The predicted error magnified by GP is considerably small, indicating that as long as the training data is reliable, the GP model will be adequate for making accurate predictions, even with only a limited number of computationally expensive simulations. 


\section{Process design map using Gaussian process model}

In this section, the process design maps of two metals, 316L and 17-4 PH stainless steel, are developed using our Gaussian process regression model. According to previous experimental and computational studies [6, 32-34], the basic criterion of identifying the keyhole mode is

$$
\frac{2 d}{w} \geq 1
$$

where $d$ is the remelted depth, $w$ is the melt pool width, and the quantity, $\frac{d}{w}$, is the normalized depth. With a reliable dataset of the normalized depth with respect to laser power and laser scan speed, the GP model is able to predict the conduction mode region where $\frac{2 d}{w}<1$, and keyhole mode where $\frac{2 d}{w} \geq 1$.

Besides this criterion, Hann et al. [35] proposed another empirical method to identify the keyhole mode. By experimental results, they first found that the normalized depth is proportional to the product of power density multiplied by the square root of the laser interaction time, such that:

$$
\frac{d}{w} \propto P_{d} \sqrt{\tau}
$$

where $P_{d}=\frac{P}{\pi \sigma^{2}}$ is the power density, and $\tau=\frac{2 \sigma}{v}$ is the laser interaction time. Then, the slope of the relationship was determined by dimensional analysis. Finally, the normalized enthalpy term is proposed to identify the keyhole mode is based, such that:

$$
\frac{\Delta H}{h_{s}}=\frac{A P}{\rho h_{s} \sqrt{\pi D v\left(\frac{\sigma}{4}\right)^{3}}}
$$

where $\Delta H$ is the specific enthalpy, $h_{s}$ is the enthalpy at melting, $A$ is the material absorptivity of the laser power, $P$ is the laser power, $\rho$ is density, $D$ is thermal diffusivity, $v$ is the laser scan speed, $\sigma$ is the laser beam size D4 $\sigma$. After that, King et al. [6] quantified a threshold valud of 30 for 316L stainless steel based on experimental study. Therefore, it predicts the occurance of keyhole mode when $\frac{\Delta H}{h_{s}} \geq 30$, and condcution mode when $\frac{\Delta H}{h_{s}}<30$. As all the involved terms in this criterion are processing parameters, it enables designers to identify the preferred conduction mode in L-PBF conveniently. However, this criterion is an empirical method, it is crucial to test it to other materials before applying it. 
In this work, two sets of experimental data of $316 \mathrm{~L}$ and 17-4 PH stainless steels will be used to train our GP model. To apply the $\frac{2 d}{w} \geq 1$ criterion, the output of the training dataset has changed from remelted depth to normalized depth, $\frac{d}{w}$. After training, GP model will then make predictions on the region of keyhole mode using the $\frac{2 d}{w} \geq 1$ criterion. The GP predicted region will then be compared against the region predicted by the normalized enthalpy criterion, thus testing the adequacy of the criterion.

\subsection{Case 1: 316L stainless steel}

The normalized enthalpy criterion of $316 \mathrm{~L}$ stainless steel is tested in this section. Table S2 in the Online Resource Electronic Supplementary Materials shows the experimental dataset from Kamath et al. [36]. Table S3 in the Online Resource Electronic Supplementary Materials shows the material properties used for the melt pool size and enthalpy calculations.

Using the trained GP model with the normalized depth computed from the dataset in Table S2, a

contour plot of the quantity, $\frac{2 d}{w}$, can be obtained, as shown in Fig. 6 (a). The preferred conduction mode region can be readily identified from Fig. 6 (a). For example, if the expected laser scan speed is kept at $1.5 \mathrm{~m} / \mathrm{s}$, then the laser power should not exceed $204 \mathrm{~W}$. Note that though the contour plot displays the entire range of laser scan speed and laser power, the predictions outside the marked boundary or extrapolated by the GP model may accompany with relatively large uncertainty and should be used with caution. 


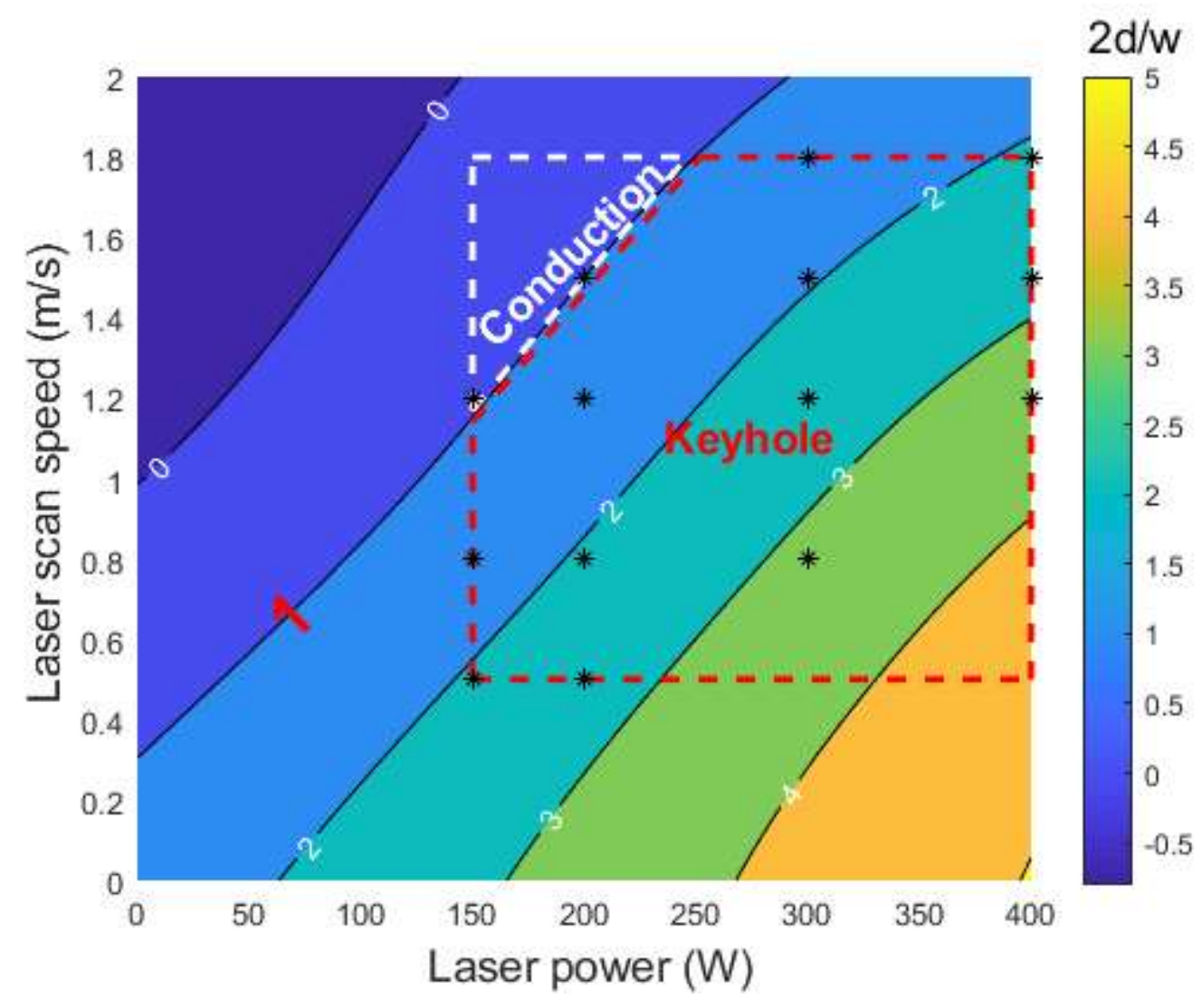

(a) 


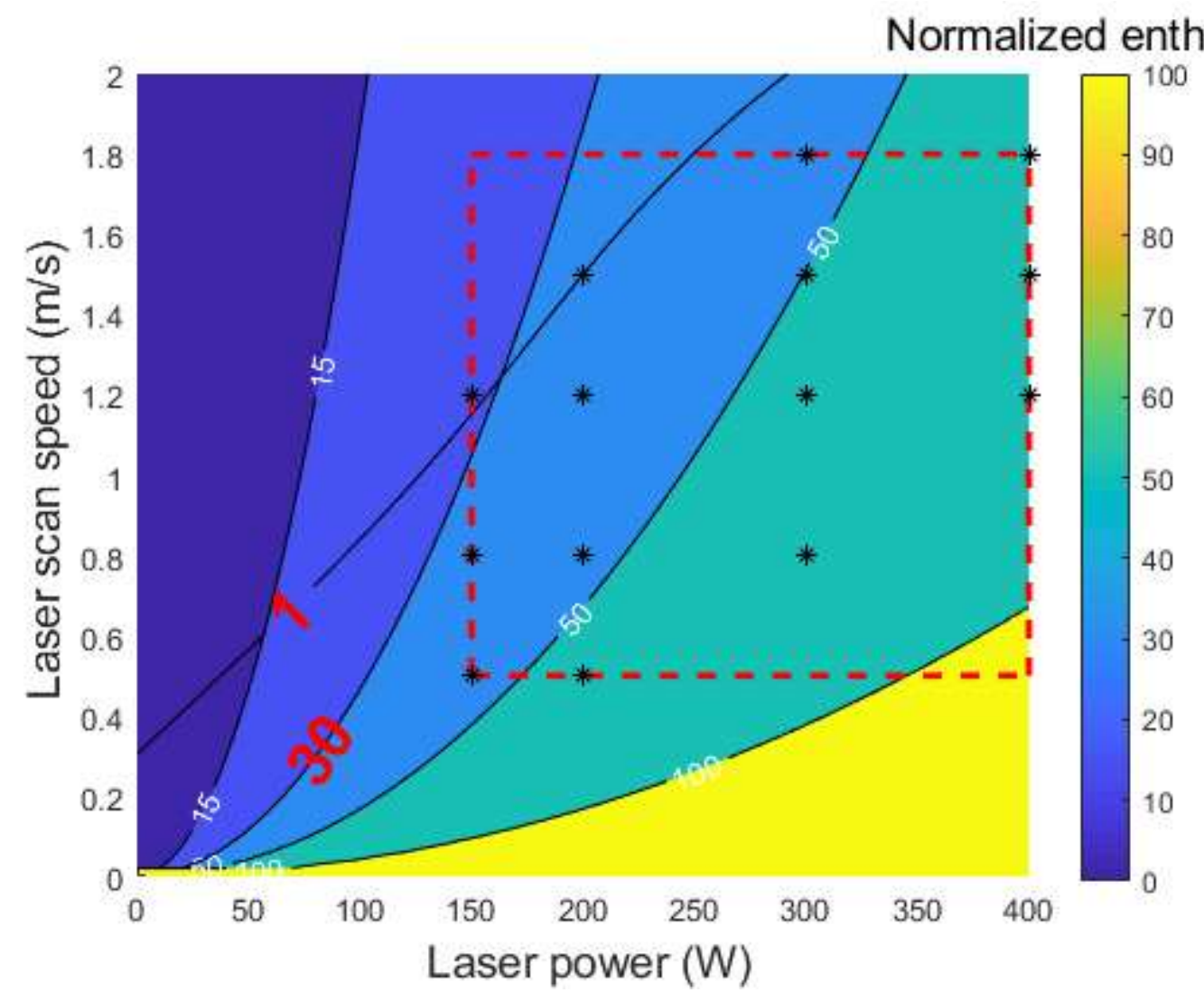

(b)

Fig. 6. Process design map of 316L stainless steel showing the keyhole mode and conduction mode regions. (a) Using $\frac{2 d}{w} \leq 1$ criterion. (b) Using normalized enthalpy criterion. The red dashed square represents the range of the laser parameters used in the experiment. In (a), the dark blue region is the preferred conduction mode region. In (b), the contour line in red text is $\frac{2 d}{w}=1$ from (a), which is to compare against the normalized enthalpy criterion. The black stars represent the experimental data.

The conduction mode region in Fig. 6 (a) is then compared against Fig. 6 (b), which is the contour plot of the normalized enthalpy calculated by Eq. (7). The boundary separating the two modes, i.e., the contour line of $\frac{2 d}{w}=1$ in Fig. 6 (a), is also plotted in Fig. 6 (b) to better compare the two criteria. Within the red dashed square region, the normalized enthalpy criterion provides acceptable predictions with a little deviation. Therefore, the empirical normalized enthalpy 
criterion is capable of distinguishing the two heat transfer modes, but additional experimental data points are still recommended when normalized enthalpy is around 30 .

It should be noted that Tapia et al. [20] also conducted a similar test to the normalized enthalpy criterion of 316L stainless steel using a GP model. In their result, the predictions made by both criteria closely matched. However, there are several differences between our work. First, the dataset they used to train their GP model contained those which used to quantify the $\frac{\Delta H}{h_{s}}=30$ criterion [6]. Therefore, the matching of both predictions verifies the validation of their GP model, but cannot confirm the accuracy of the normalized enthalpy criterion. Second, the covariance function of GP they used was the power exponential kernel, whereas in our model the Matérn 5/2 kernel is employed. While in most case the selection of kernel functions doesn't significantly affect the GP predictions, it can be a potential source of small deviation. Third, the powder layer thickness of our dataset is $30 \mu \mathrm{m}$, whereas the layer thickness of their dataset was $50 \mu \mathrm{m}$. Consequently, our result exposes that the normalized enthalpy criterion is dependent on the layer thickness, which is currently not included in its formula. Hence, the normalized enthalpy criterion for $316 \mathrm{~L}$ stainless steel is in need of calibration with the consideration of layer thickness.

\subsection{Case 2: 17-4 PH stainless steel}

This section tests the normalized enthalpy criterion of another material, 17-4 PH stainless steel. The training dataset is displayed in Table S4. Similar to the case of 316L stainless steel, the contour plot of the quantity $\frac{2 d}{w}$ is generated using the GP model trained by the dataset in Table S4 in the Online Resource Electronic Supplementary Materials, shown in Fig. 7 (a). It should be noted that in this case, the number of levels of laser power in training dataset is only two, which may result in a larger uncertainty of the predictions along the path changing the laser power. In addition, the range of the laser scan speed and laser power displayed in Fig. 7 (a) is set to be the same as the experimental dataset. 


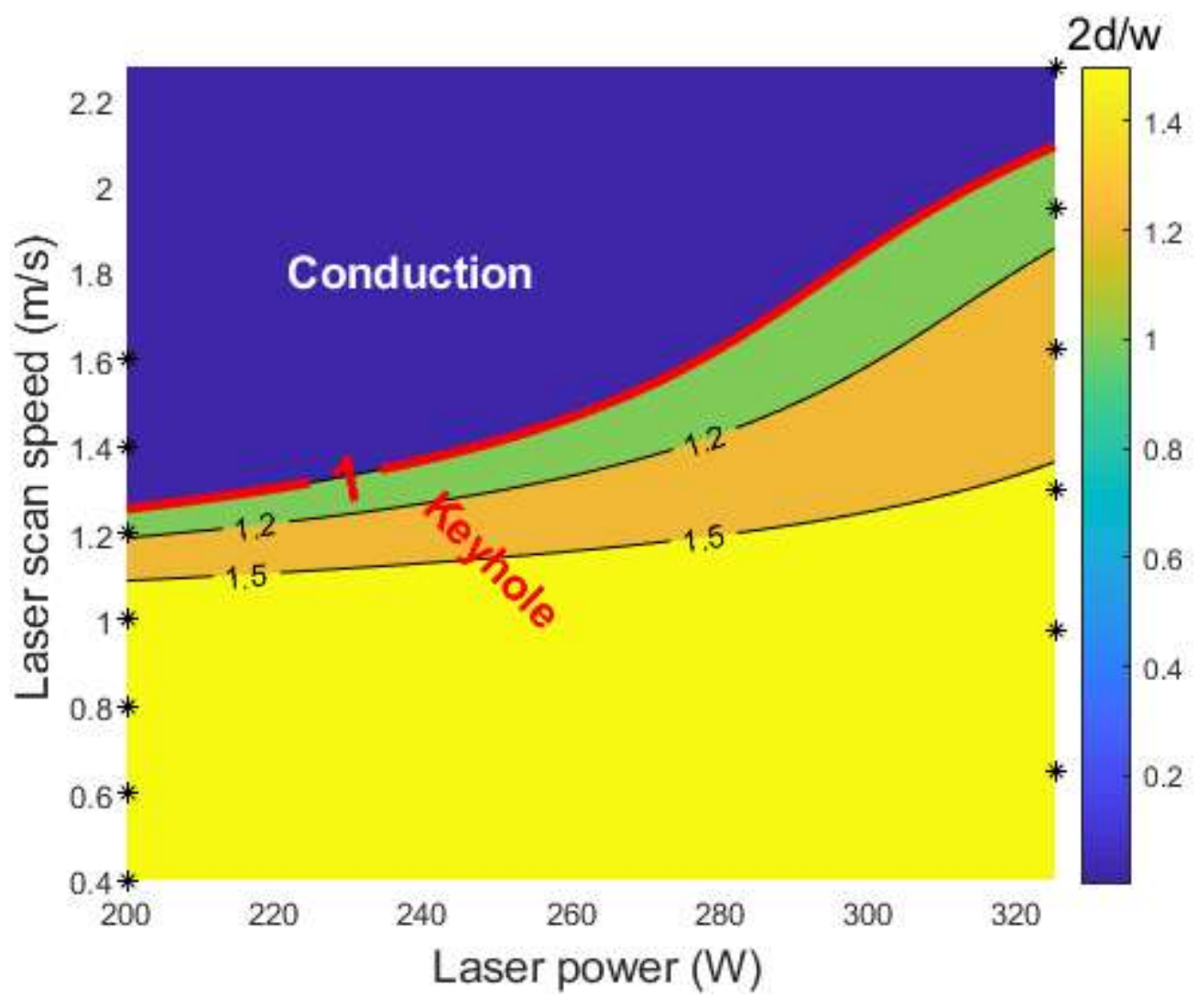

(a) 


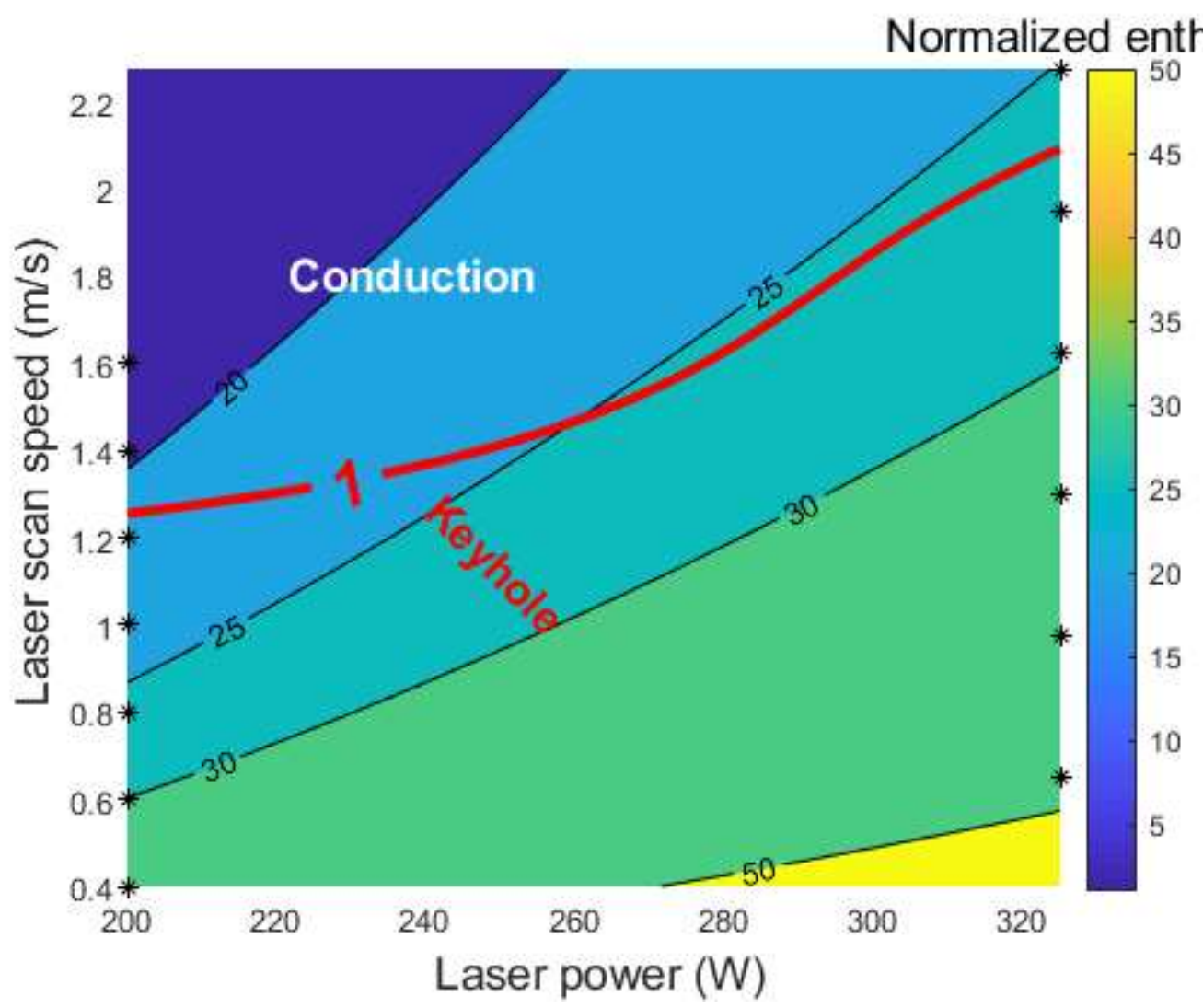

(b)

Fig. 7. Process design map of 17-4 PH stainless steel showing the keyhole mode and conduction mode regions. (a) Using $\frac{2 d}{w} \geq 1$ criterion. (b) Using normalized enthalpy criterion. In (a), the dark blue region is the preferred conduction mode region. In (b), the contour line in red is $\frac{2 d}{w}=1$ from (a), which is to compare against the normalized enthalpy criterion. The black stars represent the experimental data.

Fig. 7 (b) shows the process design map using the normalized enthalpy. It can be observed that the contour line of $\frac{2 d}{w}=1$ is close to $\frac{\Delta H}{h_{s}}=25$, indicating that the normalized enthalpy criterion should be revised to $\frac{\Delta H}{h_{s}} \geq 25$ for 17-4 PH stainless steel. It also suggests that the normalized enthalpy criterion is sensitive to the selection of the materials. Therefore, to make use of the convenient normalized enthalpy criterion, calibration is still needed when switching materials. In summary, machine learning models can be applied to process design and optimization. The 
preferred conduction mode region can be predicted by our GP model after trained by reliable datasets. Machine learning models trained by reliable experimental data will be very helpful to calibrate this criterion and thus take advantage of it.

In terms of computational costs, it is noted that when taking the training time of 480 hours (total 24 simulations and 20 hours for each described in Section 3.1) into consideration, the total time for training and application of the GP model will be more than 480 hours, instead of 1 minute. However, GP can literally avoid further observation costs and time, no matter the how many new cases to be predicted in the future. Without GP, any additional observations made by either experimental or computational investigations will need additional costs and time.

Additionally, in this work, the GP is applied as a regression method, which is different from an interpolation method. For interpolation, the goal is to find a function of a predefined form (e.g., polynomial, spline) that has the values in that points exactly as specified. This means that the function must pass through all the data points. In comparison, for regression, the goal is find a function that minimizes some cost, usually sum of squares of errors. The function doesn't have to have the exact values at given points. A good approximation is provided.

\section{Conclusions}

In this study, a Gaussian process regression model is developed to assist the process design and optimization of the L-PBF additive manufacturing process. The major findings are summarized as follow:

1. The predictions made by the trained GP model are in good agreement with the experimental observations. With a limited number (24) of data points, the mean absolute prediction error is only magnified by $0.6 \mu \mathrm{m}$ using the GP model for a powder layer thickness of $30 \mu \mathrm{m}$, and can be further decreased by obtaining more training data.

2. The process design maps of two metals, 316L and 17-4 PH stainless steel, are developed using our trained Gaussian process regression model. The preferred conduction mode regions of laser power and laser scan speed for 316L and 17-4 PH stainless steel are predicted by the GP model trained by experimental datasets using the $\frac{2 d}{w}<1$ criterion. 
3. The normalized enthalpy criterion of identifying keyhole mode is evaluated for both 316L and 17-4 PH stainless steel. For 316L, the result suggests that the $\frac{\Delta H}{h_{s}} \geq 30$ criterion should be related to the powder layer thickness. For 17-4 PH, the criterion should be revised to $\frac{\Delta H}{h_{s}} \geq 25$.

\section{Acknowledgements}

The authors acknowledge the support provided by the National Science Foundation (No.1836555), Walmart Foundation (project title: Optimal Plastic Injection Molding Tooling Design and Production through Advanced Additive Manufacturing), and Praxair's TruForm ${ }^{\mathrm{TM}}$ AMbition Grant awarded to Indiana University-Purdue University Indianapolis. 


\section{References}

[1] G. Tapia and A. Elwany, J. Eng. Ind., 136, 6, 060801 (2014).

[2] R. Rai, J. Elmer, T. Palmer, and T. DebRoy, J. Phys. D: Appl. Phys., 40, 18, 5753 (2007).

[3] J. D. Madison and L. K. Aagesen, Scr. Mater., 67, 9, 783 (2012).

[4] S. Bontha, N. W. Klingbeil, P. A. Kobryn, and H. L. Fraser, J. Mater. Process. Technol., 178, 1-3, 135 (2006).

[5] C. Kamath, Int. J. Adv. Manuf. Techno., 86, 5-8, 1659 (2016).

[6] Wayne E. King, Holly, D. Barth, Victor M. Castillo, Gilbert F. Gallegos, John W. Gibbs, Douglas E. Hahn, Chandrika Kamath, and Alexander M. Rubenchik, J. Mater. Process. Technol, 214, 12, 2915 (2014).

[7] M. Yan and P. Yu, in Sintering techniques of materials: InTech (2015) doi: $10.5772 / 59275$.

[8] Qilin Guo, Cang Zhao, Luis I. Escano, Zachary Young, Lianghua Xiong, Kamel Fezzaa, Wes Everhart, Ben Brownd, Tao Sun, Lianyi Chen, Acta Mater., 151, 169 (2018).

[9] A. Gusarov, I. Yadroitsev, P. Bertrand, and I. Smurov, J. Heat Transfer, 131, 7, 072101 (2009).

[10] Chinnapat Panwisawas, Chunlei Qiu, Magnus J. Anderson, Yogesh Sovani, Richard P. Turner, Moataz M. Attallah. Jeffery W. Brooks, Hector C. Basoalto, Comput. Mater. Sci., 126, 479 (2017).

[11] J. Zhang, Y. Zhang, W. H. Lee, L. Wu, H.-H. Choi, and Y.-G. Jung, Met. Powder Rep., (2018) doi: 10.1016/j.mprp.2018.01.003.

[12] Y. Zhang and J. Zhang, J. Mater. Res., 31, 15, 2233 (2016).

[13] Y. Zhang and J. Zhang, Addit. Manuf., 16, 49 (2017).

[14] Y. Zhang, X. Xiao, and J. Zhang, Results Phys., 13, 102336 (2019).

[15] J. Zhang, L. Wu, Y. Zhang, and L. Meng, Met. Powder Rep., 74, 1, 20 (2019).

[16] Y. Zhang and J. Zhang, Addit. Manuf., 28, 750 (2019).

[17] E. Alpaydin, Introduction to machine learning, 2nd ed. (London, England: the MIT press, 2009), pp. 4-16.

[18] Z. Zhu, N. Anwer, Q. Huang, and L. Mathieu, CIRP Ann., (2018) doi:

10.1016/j.cirp.2018.04.119.

[19] G. Tapia, A. Elwany, and H. Sang, Addit. Manuf., 12, 282 (2016).

[20] G. Tapia, S. Khairallah, M. Matthews, W. E. King, and A. Elwany, Int. J. Adv. Manuf. Techno., 94, 9-12, 3591 (2018).

[21] F. Caiazzo and A. Caggiano, Mater., 11, 3, 444 (2018).

[22] J. Zhang, P. Wang, and R. X. Gao, Comput. Ind., 107, 11 (2019).

[23] Mojtaba Mozaffar, Arindam Paul, Reda Al-Bahrani, Sarah Wolff, Alok Choudhary, Ankit Agrawal, Kornel Ehmann, Jian Cao, Manuf. Lett., 18, 35 (2018).

[24] S. Haykin, Neural networks: a comprehensive foundation, 1st ed. (Upper Saddle River, NJ, USA: Prentice Hall PTR, 2009).

[25] C. K. Williams and C. E. Rasmussen, Gaussian processes for machine learning, 1st ed. (London, England: the MIT press, 2016).

[26] GPy, "GPy: A gaussian process framework in python", https://sheffieldml.github.io/GPy/. (accessed July 1, 2019)

[27] S. A. Khairallah, A. T. Anderson, A. Rubenchik, and W. E. King, Acta Mater., 108, 36 (2016).

[28] I. Choquet, A. J. Shirvan, and H. Nilsson, J. Phys. D: Appl. Phys., 45, 20, 205203 (2012). 
[29] M. Courtois, M. Carin, P. Le Masson, S. Gaied, and M. Balabane, J. Phys. D: Appl. Phys., 46, 50, 505305 (2013).

[30] W. Tan, N. S. Bailey, and Y. C. Shin, J. Phys. D: Appl. Phys., 46, 5, 055501 (2013).

[31] I. Yadroitsev, P. Krakhmalev, I. Yadroitsava, S. Johansson, and I. Smurov, J. Mater. Process. Technol., 213, 4, 606 (2013).

[32] T. Eagar and N. Tsai, Weld. J., 62, 12, 346 (1983).

[33] V.-P. Matilainen, H. Piili, A. Salminen, and O. Nyrhilä, Phys. Procedia, 78, 377 (2015).

[34] Y. Zhang, "Multi-Scale Multi-Physics Modeling Of Laser Powder Bed Fusion Process Of Metallic Materials With Experiment Validation", PhD thesis, Purdue University, 2018

[35] D. Hann, J. Iammi, and J. Folkes, J. Phys. D: Appl. Phys., 44, 44, 445401 (2011).

[36] C. Kamath, B. El-dasher, G. F. Gallegos, W. E. King, and A. Sisto, Int. J. Adv. Manuf. Techno., 74, 1-4, 65 (2014).

[37] I. Fieldhouse and J. Lang, "Measurement of thermal properties, 1st ed. (Chicago, IL, USA: Armour Research Foundation, 1961). 